\title{
Microwave-Assisted Synthesis of Indole-2-Carboxylic Acid Esters in Ionic Liquid
}

\author{
Lijun $\mathrm{Gu}^{*, a}$ and Xiangguang $\mathrm{Li}^{b}$ \\ ${ }^{a}$ Key Laboratory of Ethnic Medicine Resource Chemistry, Yunnan University of Nationalities, \\ State Ethnic Affairs Commission \& Ministry of Education of P. R. China, Kunming, 650031, China \\ ${ }^{b}$ Department of Chemistry, Northwest University, Xi'an, China
}

\begin{abstract}
Um procedimento melhorado para a síntese de ésteres ácido indol-2-acético em rendimentos excelentes foi obtido pela condensação de 2-halo aril aldeídos ou cetonas e isocianoacetato de etila usando líquido iônico sob irradiação de microondas controlada $(100 \mathrm{~W})$ a $50{ }^{\circ} \mathrm{C}$. Este método oferece um número de vantagens em termos de metodologia simples, com rendimento elevado num tempo de reação curto e em condições de reação amenas.
\end{abstract}

\begin{abstract}
An improved procedure for the synthesis of indole-2-carboxylic acid esters in excellent yields has been achieved by the condensation of 2-halo aryl aldehydes or ketones and ethyl isocyanoacetate using ionic liquid under controlled microwave irradiation $(100 \mathrm{~W})$ at $50{ }^{\circ} \mathrm{C}$. This method offers a number of advantages in terms of methodology, high-product yield, short period of conversion, mild reaction conditions and easy workup.
\end{abstract}

Keywords: indole, synthesis, ionic liquid, microwave irradiation

\section{Introduction}

The indole moiety is a vital structural unit found in a large array of natural products and pharmaceuticals. Consequently, synthetic approaches toward indoles have attracted significant attention..$^{1-3}$ In many cases, the wellstudied Fischer indole synthesis is the method of choice, but this approach suffers from problems such as low yield and formation of side products. ${ }^{4}$ In our design of new inhibitors of aspartyl protease, ${ }^{5,6}$ we were interested in differently substituted indole-2-carboxylic acid esters. Owing to their structural diversity and remarkable biological functions, a multitude of methods exist for the synthesis of these compounds. The conventional method is the Hemetsberger-Knittel indole synthesis. ${ }^{7}$ This method involves the condensation between an arylaldehyde and an azidoacetate to provide $a$-azidocinnamates which upon heating give indoles. One problem is associated with employing high boiling solvents such as mesitylene, xylene and toluene. ${ }^{8,9}$ In addition, the yield of the reaction is low. ${ }^{10} \mathrm{Cai}$ and co-workers ${ }^{11}$ developed a copper-catalyzed cascade process from 2-halo aryl aldehydes/ketones with isocyanoacetate for synthesis of these derivatives. However, the procedure has some drawbacks such as long reaction

*e-mail: gulijun2005@126.com time and moderate yields. For the cyclization of 2-chloro aryl aldehydes/ketones with isocyanoacetate, the yield of the reaction is unsatisfactory and the temperature of the reaction is high. Therefore, it is necessary to develop a more practical method for synthesis of such a significant scaffold.

The research and application of green chemistry principles have led to the development of cleaner processes. Since the start of this century an ever-growing number of studies has been published describing the use of ionic liquids. ${ }^{12-17}$ Microwave-assisted organic synthesis has shown to be a valuable tool for reducing reaction times, getting cleaner reactions, improving yields, simplifying workup and designing energy-saving protocols. ${ }^{18,19}$

Due to their ionic nature, ionic liquids allow highly effective interactions with microwave energy for the rapid generation of products with generally high yields. ${ }^{20}$ Herein, we developed a fast and practical method for the synthesis of indole-2-carboxylic acid esters in the presence of 1-methyl-3-butylimidazolium hydroxide ( [bmim $] \mathrm{OH}$ ) under controlled microwave irradiation $(100 \mathrm{~W})$ at $50^{\circ} \mathrm{C}$. The synthetic sequence is depicted in Scheme 1.

\section{Results and Discussion}

Initial studies were carried out using 2-bromo benzenealdehyde as the substrate and isocyanoacetate as 


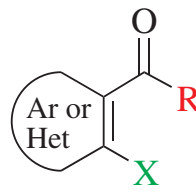

1

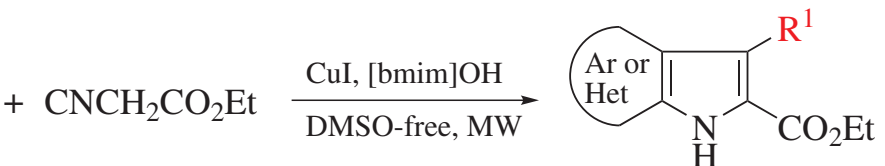

2

$$
\mathrm{R}^{1}=\mathrm{H} \text { or } \mathrm{Me} \quad \mathrm{X}=\mathrm{I}, \mathrm{Br} \text { or } \mathrm{Cl}
$$

Scheme 1. Sequence for the synthesis of indole-2-carboxylic acid esters in the presence of 1-methyl-3-butylimidazolium hydroxide ([bmim]OH ) under controlled microwave irradiation.

the nitrogen source in [bmim] $\mathrm{OH}$ under the catalysis of $\mathrm{CuI}$ (12 mol\%) for $8 \mathrm{~h}$, the desired product ethyl $1 H$-indole2 -carboxylate was obtained in moderate yield $(57 \%)$. When the reaction was irradiated under controlled microwave $\left(100 \mathrm{~W}\right.$ ) for $10 \mathrm{~min}$ at $50{ }^{\circ} \mathrm{C}, 91 \%$ of the desired product was reached. Encouraged by the result, other $\mathrm{Cu}$ reagents, such as $\mathrm{CuBr}$ or $\mathrm{CuCl}$, were investigated in the presence of [bmim] $\mathrm{OH}$ under microwave irradiation. The results showed that replacement of $\mathrm{CuI}$ with $\mathrm{CuBr}$ slightly affected the outcome of this reaction (entry 1 and entry 9, Table 1). $\mathrm{CuCl}$ was less active (entries 9-10, Table 1). When other copper sources such as $\mathrm{Cu}_{2} \mathrm{O}$ and $\mathrm{Cu}(\mathrm{OAc})_{2}$ were used, no desired product was detected.

Next, the reaction time and the temperature were investigated in order to find the optimal reaction conditions. It was found that $10 \mathrm{~min}$ was long enough to run the reaction. Raising the reaction temperature did not obviously improve the reaction yield (entries 11-12, Table 1). Also an extended reaction time has no effect on the conversion. Finally, the optimal conditions for parallel synthesis were determined to be $12 \mathrm{~mol} \% \mathrm{CuI}$ at $50{ }^{\circ} \mathrm{C}$ for $10 \mathrm{~min}$. The substrate variation was then investigated. To our satisfaction, the reaction shows a wide scope for the structural variations of aryl aldehydes or ketones under the optimized reaction conditions. Both electron-donating and electron-withdrawing groups allowed smooth transformation of aryl aldehydes or ketones into the corresponding products with high yields. Performing the reaction with 2-chloro aryl aldehydes or ketones gave good yields (entries 13-16, Table 1). It was noteworthy that hetero-aryl aldehydes or ketones with varied substitutions were found to undergo the reaction (entries 17-20, Table 1). It was found that DMSO-free condition gave the corresponding product in excellent yield (entries 21-24, Table 1). To illustrate the preparative utility, our procedure was performed on a large scale (entry 25, Table 1). A 60-mmol-scale synthesis gave the desired product in $88 \%$ yield.

\section{Conclusions}

In summary, in this study we have successfully extended the use of ionic liquid [bmim] $\mathrm{OH}$ under microwave irradiation for assembly of indole-2-carboxylic acid esters in high yields. The experimental procedure is very simple and the reaction time is short. The synthetic protocols described above have proven amenable to scale-up to over $10 \mathrm{-g}$ quantities.

\section{Experimental}

\section{General}

Reagents were obtained commercially and used as received. Solvents were purified and dried by standard methods. [bmim]OH was synthesized according to the method described in the literature. ${ }^{21}$ Microwave reactions were performed on a CEM Explorer Hybrid 12 Discover, with built in temperature/pressure probes and associated software. The melting points (mp) were determined on an XT-4 micro melting point apparatus and uncorrected. Infrared (IR) spectra were recorded on an EQUINOX-55 spectrometer on a $\mathrm{KBr}$ matrix. ${ }^{1} \mathrm{H}$ nuclear magnetic resonance (NMR) spectra were recorded on an INOVA-400 NMR spectrometer using TMS as an internal standard. Chemical shift values $(\delta)$ are given in $\mathrm{ppm}$. Elemental analyses were performed on a Vario EL III CHNS analyzer. Electrospray mass spectra were obtained with an MALDI-TOF Mass spectrometer. 200-300 mesh silica gel was used for column chromatography.

\section{Representative procedure for the synthesis of 2}

An oven-dried 10-mL microwave vial was charged with copper(I) iodide $(0.24 \mathrm{mmol})$, [bmim] OH $(2 \mathrm{~mL})$, aldehydes or ketones $(1 \mathrm{mmol})$, ethyl isocyanoacetate $(1.1 \mathrm{mmol})$, DMSO $(1 \mathrm{~mL})$, or DMSO-free with the addition of a stirred bar. The reaction vessel was sealed, evacuated and flushed with argon three times. The mixture was irradiated by $100 \mathrm{~W}$ microwave at $50{ }^{\circ} \mathrm{C}$ for $10 \mathrm{~min}$. The reaction mixture was partitioned between ethyl acetate and water. The organic layers were washed with water and brine, dried over anhydrous $\mathrm{MgSO}_{4}$ and concentrated under vacuum to yield the crude product. The crude product 
Table 1. Reactions of 2-halo aryl aldehydes or ketones with ethyl isocyanoacetate ${ }^{\text {a }}$

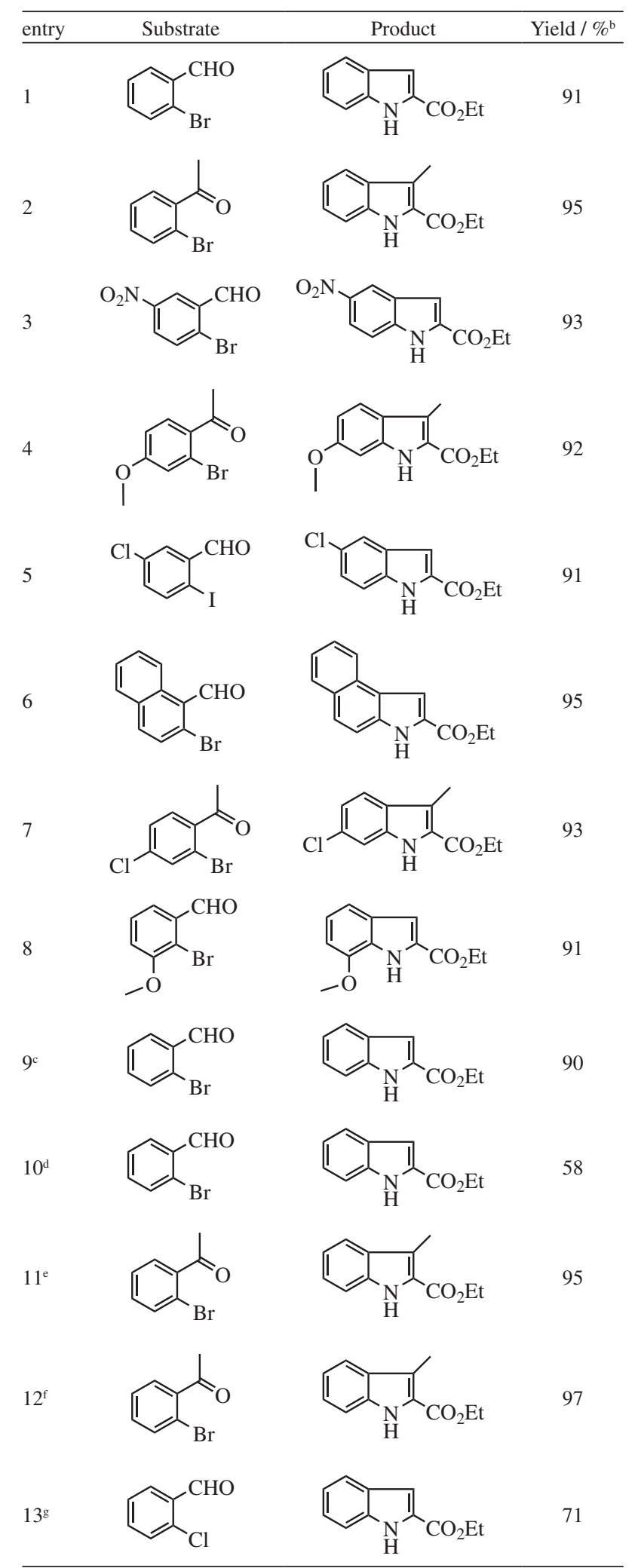

entry Substrate Product

$16^{\mathrm{g}}$<smiles>CC(=O)c1sccc1Cl</smiles>

17<smiles>O=Cc1ncccc1Br</smiles>

18<smiles>O=Cc1sccc1Br</smiles>

19<smiles>CC(=O)c1sccc1Br</smiles><smiles>[101In]</smiles><smiles>O=Cc1sc([N+](=O)[O-])cc1Br</smiles><smiles>CCOC(=O)c1cc2sc([N+](=O)[O-])cc2[nH]1</smiles>

$21^{\mathrm{h}}$<smiles>O=Cc1ccccc1Br</smiles>
$22^{\mathrm{h}}$<smiles>CC(=O)c1ccccc1Br</smiles><smiles>[111In]</smiles><smiles>O=Cc1ncccc1Br</smiles><smiles>[14CH3]</smiles><smiles>CC(=O)c1sccc1Br</smiles>
$25^{\mathrm{i}}$<smiles>O=Cc1ccccc1Br</smiles><smiles>CCOC(=O)c1cc2ccccc2[nH]1</smiles><smiles>CCOC(=O)c1[nH]c2ccsc2c1C</smiles><smiles>CCOC(=O)c1cc2ncccc2[nH]1</smiles><smiles>CCOC(=O)c1cc2sccc2[nH]1</smiles><smiles>CCOC(=O)c1[nH]c2ccsc2c1C</smiles><smiles>CCOC(=O)c1[nH]c2ccccc2c1C</smiles><smiles>CCOC(=O)c1[nH]c2ccsc2c1C</smiles><smiles>CCOC(=O)c1cc2ccccc2[nH]1</smiles>

aReaction conditions: $\mathrm{CuI}(0.24 \mathrm{mmol})$, [bmim] OH ( $2 \mathrm{~mL})$, aryl bromides $(1 \mathrm{mmol})$, ethyl isocyanoacetate (1.1 mmol), DMSO (1 mL), Ar atmosphere,

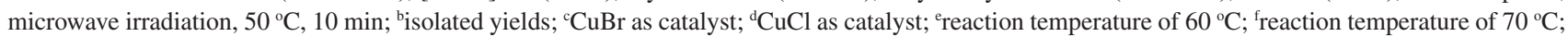
${ }^{g}$ reaction temperature of $60{ }^{\circ} \mathrm{C}$; ${ }^{\mathrm{h}}$ without DMSO; ${ }^{\mathrm{i}} \mathrm{CuI}(15 \mathrm{mmol}),[\mathrm{bmim}] \mathrm{OH}(35 \mathrm{~mL})$, aryl bromides $(60 \mathrm{mmol})$, ethyl isocyanoacetate $(66 \mathrm{mmol}), \mathrm{Ar}$ atmosphere, microwave irradiation, $50^{\circ} \mathrm{C}$. 
was purified by flash chromatography with ethyl acetate/ petroleum ether as eluent on silica gel to afford the desired product.

\section{Supplementary Information}

Supplementary data are available free of charge at http://jbcs.sbq.org.br as PDF file.

\section{Acknowledgments}

The authors thank the Youth Fund of Yunnan University of Nationalities (No. 09QN04) for finacial support. We thank Mr. Ahmad for reproducing the results presented in entries 1 and 19 of Table 1.

\section{References}

1. Humphrey, G. R.; Kuethe, J. T.; Chem. Rev. 2006, 106, 2875.

2. Bandini, M.; Eichholzer, A.; Angew. Chem., Int. Ed. 2009, 48, 9608.

3. Sharma, V.; Kumar, P.; Pathak, D.; J. Heterocycl. Chem. 2010, 47, 491.

4. Miyata, O.; Kimura, Y.; Muroya, K.; Hiramatsu, H.; Naito, T.; Tetrahedron Lett. 1999, 40, 3601.

5. Gu, L. J.; Liu, F. L.; Li, X. G.; Gao, B.; Yang, B. Q.; Synth. Commun. 2010, 40, 242.

6. Gu, L. J.; Yang, B. Q.; Liu, F. L.; J. Braz. Chem. Soc. 2010, 21, 58.

7. Knittel, D.; Synthesis 1985, 2, 186.

8. Hickey, D. M. B.; MacKenzie, A. R.; Moody, C. J.; Rees, C. W.; J. Chem. Soc., Perkin Trans. 1 1987, 921.
9. Secchi, M.; Derudas, M.; Dallochio, R.; Dessi, A.; Bacchi, A.; Sannia, L.; Carta, F.; Palomba, M.; Ragab, O.; Chan, C.; Shoemaker, R.; Sei, S.; Dayam, R.; Neamati, N.; J.Med. Chem. 2004, 47, 5298.

10. Venable, J. D.; Cai, H.; Chai, W.; Dvorak, C. A.; Grice, C. A.; Jablonowski, J. A.; Shah, C. R.; Kwok, A. K.; Ly, K. S.; Pio, B.; Wei, J.; Desai, P. J.; Jiang, W.; Nguyen, S.; Ling, P.; Wilson, S. J.; Dunford, P. J.; Thurmond, R. L.; Lovenberg, T. W.; Karlsson, L.; Carruthers, N. I.; Edwards, J. P.; J. Med. Chem. 2004, 48, 8289.

11. Cai, Q.; Li, Z. Q.; Wei, J. J.; Ha, C. Y.; Pei, D. Q.; Ding, K.; Chem. Commun. 2009, 46, 7581.

12. Lovelock, K. R.; Villar-Garcia, I. J.; Maier, F.; Steinrück, H.; Licence, P.; Chem. Rev. 2010, 111, 5158.

13. Pilissão, C.; Carvalho, P. O.; Nascimento, M. G.; J. Braz. Chem. Soc. 2010, 21, 973.

14. Polshettiwar, V.; Varma, R. S.; Acc. Chem. Res. 2008, 41, 629.

15. Pastre, J. C.; Génisson, Y.; Saffon, N.; Dandurand, J.; Correia, C. R. D.; J. Braz. Chem. Soc. 2010, 21, 821.

16. Dupont, J.; J. Braz. Chem. Soc. 2004, 15, 341.

17. Lenardão, E. J.; Gonçalves, L. C. C.; Mendes, S. R.; Saraiva, M. T.; Alves, D.; Jacob, R. J.; Perin, G.; J. Braz. Chem. Soc. 2010, 21, 2093.

18. Souza, R. O. M. A.; Quim. Nova 2011, 34, 497.

19. Wang, X. Q.; Zhao, Z. G.; Li, W. J.; Liu, X. L.; Han, T.; Chin. J. Org. Chem. 2010, 30, 764.

20. Hoffmann, H.; Nuchter, M.; Ondruschka, B.; Wasserscheid, P.; Green Chem. 2003, 5, 296.

21. Ranu, B. C.; Banerjee, S.; Org. Lett. 2005, 7, 3049.

Submitted: May 17, 2011

Published online: August 25, 2011 


\title{
Microwave-Assisted Synthesis of Indole-2-Carboxylic Acid Esters in Ionic Liquid
}

\author{
Lijun $\mathrm{Gu}^{*, a}$ and Xiangguang $\mathrm{Li}^{b}$ \\ ${ }^{a}$ Key Laboratory of Ethnic Medicine Resource Chemistry, Yunnan University of Nationalities, \\ State Ethnic Affairs Commission \& Ministry of Education of P. R. China, Kunming, 650031, China \\ ${ }^{b}$ Department of Chemistry, Northwest University, Xi'an, China
}

Ethyl 1H-indole-2-carboxylate

White solid; mp 120-122 ${ }^{\circ} \mathrm{C}$ (lit. ${ }^{1} \mathrm{mp} 121-123^{\circ} \mathrm{C}$ ); IR (KBr) $v_{\text {max }} / \mathrm{cm}^{-1} 3463,3431,2929,1711$ and $1695 ;{ }^{1} \mathrm{H} \mathrm{NMR}$ $\left(\mathrm{CDCl}_{3}, 400 \mathrm{MHz}\right) \delta 1.41(\mathrm{t}, 3 \mathrm{H}, J 6.8 \mathrm{~Hz}), 4.39(\mathrm{q}, 2 \mathrm{H}$, $J 6.8 \mathrm{~Hz}), 7.23-7.16(\mathrm{~m}, 1 \mathrm{H}), 7.29-7.33(\mathrm{~m}, 2 \mathrm{H}), 7.49-7.56$ $(\mathrm{m}, 1 \mathrm{H}), 7.69(\mathrm{~d}, 1 \mathrm{H}, J 9.6 \mathrm{~Hz}), 9.41(\mathrm{br} \mathrm{s}, 1 \mathrm{H}) ;{ }^{13} \mathrm{C} \mathrm{NMR}$ $\left(\mathrm{CDCl}_{3}, 75 \mathrm{MHz}\right) \delta 161.7,135.4,128.2,126.5,122.6$, 121.7, 121.1, 110.6, 109.2, 62.3, 14.7; MALDI-TOF MS $\mathrm{m} / \mathrm{z} 189\left(\mathrm{M}^{+}\right)$; Anal. calcd. for $\mathrm{C}_{11} \mathrm{H}_{11} \mathrm{NO}_{2}$ : $\mathrm{C} 69.83, \mathrm{H} 5.86$, N 7.40; found: C 70.01, H 5.69, N 7.21.

Ethyl 3-methyl-1H-indole-2-carboxylate

White solid; mp 132-134 ${ }^{\circ} \mathrm{C}$ (lit. ${ }^{2} \mathrm{mp} 134-136{ }^{\circ} \mathrm{C}$ ); IR (KBr) $v_{\max } / \mathrm{cm}^{-1} 3466,3429,2928,1716$ and 1691; ${ }^{1} \mathrm{H}$ NMR $\left(\mathrm{CDCl}_{3}, 400 \mathrm{MHz}\right) \delta 1.43(\mathrm{t}, 3 \mathrm{H}, J 9.6 \mathrm{~Hz}), 2.61$ (s, 3H), 4.33 (q, 2H, J 9.6 Hz), 7.21-7.25 (m, 1H), 7.31-7.39 $(\mathrm{m}, 2 \mathrm{H}), 7.61(\mathrm{~d}, 1 \mathrm{H}, J 8.0 \mathrm{~Hz}), 9.01(\mathrm{br} \mathrm{s}, 1 \mathrm{H}) ;{ }^{13} \mathrm{C} \mathrm{NMR}$ $\left(\mathrm{CDCl}_{3}, 75 \mathrm{MHz}\right) \delta 162.3,136.1,128.7,125.6,123.7,121.3$, $120.4,119.2,110.5,61.2,14.8,10.3$; MALDI-TOF MS m/z $203\left(\mathrm{M}^{+}\right)$; Anal. calcd. for $\mathrm{C}_{12} \mathrm{H}_{13} \mathrm{NO}_{2}$ : C 70.92, H 6.45, N 6.89; found: C 71.14, H 6.49, N 6.92.

\section{Ethyl 5-nitrol-1H-indole-2-carboxylate}

Yellow solid; mp 221-223 ${ }^{\circ} \mathrm{C}$ (lit. ${ }^{3} \mathrm{mp} 220-222{ }^{\circ} \mathrm{C}$ ); IR (KBr) $v_{\max } / \mathrm{cm}^{-1} 3460,3431,2931,1506$ and 1341; ${ }^{1} \mathrm{H}$ NMR $\left(\mathrm{CDCl}_{3}, 400 \mathrm{MHz}\right) \delta 1.39$ (t, 3H, $\left.J 4.0 \mathrm{~Hz}\right), 4.38$ $(\mathrm{q}, 2 \mathrm{H}, J 4.0 \mathrm{~Hz}), 7.41(\mathrm{~s}, 1 \mathrm{H}), 7.59$ (d, 1H, J 4.0 Hz), 8.11 $(\mathrm{d}, 1 \mathrm{H}, J 8.0 \mathrm{~Hz}), 8.63(\mathrm{~s}, 1 \mathrm{H}), 10.11(\mathrm{br} \mathrm{s}, 1 \mathrm{H}) ;{ }^{13} \mathrm{C} \mathrm{NMR}$ $\left(\mathrm{CDCl}_{3}, 75 \mathrm{MHz}\right) \delta 161.4,146.3,138.9,131.1,125.8$, 120.7, 119.5, 112.2, 110.6, 61.7, 14.3; MALDI-TOF MS $\mathrm{m} / \mathrm{z} 234\left(\mathrm{M}^{+}\right)$; Anal. calcd. for $\mathrm{C}_{11} \mathrm{H}_{10} \mathrm{~N}_{2} \mathrm{O}_{4}: \mathrm{C} 56.41, \mathrm{H} 4.30$, N 11.96; found: C 56.53, H 4.39, N 11.74.

Ethyl 6-methoxyl-3-methyl-1H-indole-2-carboxylate

Brown solid; mp 121-123 ${ }^{\circ} \mathrm{C}$ (lit. ${ }^{4} \mathrm{mp} 122-124{ }^{\circ} \mathrm{C}$ ); IR (KBr) $v_{\max } / \mathrm{cm}^{-1} 3467,3336,2990,1706$ and 1681;

*e-mail: gulijun2005@126.com
${ }^{1} \mathrm{H}$ NMR $\left(\mathrm{CDCl}_{3}, 400 \mathrm{MHz}\right) \delta 1.40$ (t, 3H, J $\left.7.2 \mathrm{~Hz}\right), 2.59$ (s, 3H), $3.81(\mathrm{~s}, 3 \mathrm{H}), 4.42(\mathrm{q}, 2 \mathrm{H}, J 7.2 \mathrm{~Hz}), 6.83(\mathrm{~d}, 1 \mathrm{H}$, $J 2.4 \mathrm{~Hz}), 6.91-7.03(\mathrm{~m}, 1 \mathrm{H}), 7.49(\mathrm{~d}, 1 \mathrm{H}, J 8.0 \mathrm{~Hz}), 9.06$ (br s, $1 \mathrm{H}) ;{ }^{13} \mathrm{C}$ NMR $\left(\mathrm{CDCl}_{3}, 75 \mathrm{MHz}\right) \delta 160.8,144.1$, 130.6, 128.4, 127.0, 121.3, 118.5 109.7, 105.2, 61.7, 56.2, 14.1, 11.6; MALDI-TOF MS m/z $233\left(\mathrm{M}^{+}\right)$; Anal. calcd. for $\mathrm{C}_{13} \mathrm{H}_{15} \mathrm{NO}_{3}$ : C 66.94, $\mathrm{H} 6.48, \mathrm{~N}$ 6.00; found: C 66.81, H 6.44, N 6.03.

Ethyl 5-chloro-1H-indole-2-carboxylate

White solid; mp 169-171 ${ }^{\circ} \mathrm{C}$ (lit. ${ }^{1} \mathrm{mp} 167-169{ }^{\circ} \mathrm{C}$ ); IR (KBr) $v_{\max } / \mathrm{cm}^{-1} 3461,3343,2928,1715$ and 1693; ${ }^{1} \mathrm{H} \mathrm{NMR}\left(\mathrm{CDCl}_{3}, 400 \mathrm{MHz}\right) \delta 1.39$ (t, 3H, J $\left.4.8 \mathrm{~Hz}\right), 4.41$ (q, 2H, J 4.8 Hz), $7.31(\mathrm{~s}, 1 \mathrm{H}), 7.41-7.44(\mathrm{~m}, 1 \mathrm{H}), 7.53$ (d, 1H, J 3.2 Hz), 7.67 (s, 1H), 9.89 (br s, 1H); ${ }^{13} \mathrm{C}$ NMR $\left(\mathrm{CDCl}_{3}, 75 \mathrm{MHz}\right) \delta 160.9,137.1,129.4,128.6,127.2$, 122.8, 120.7, 111.5, 109.3, 62.4, 14.5; MALDI-TOF MS $\mathrm{m} / z 223\left(\mathrm{M}^{+}\right)$; Anal. calcd. for $\mathrm{C}_{11} \mathrm{H}_{10} \mathrm{ClNO}_{2}$ : C 59.07, H 4.51, N 6.26; found: C 59.16, H 4.57, N 6.32.

Ethyl 3H-benz[e]indole-2-carboxylate

Brown solid; mp 164-166 ${ }^{\circ} \mathrm{C}$ (lit. ${ }^{5} \mathrm{mp} 164-165{ }^{\circ} \mathrm{C}$ ); IR (KBr) $v_{\max } / \mathrm{cm}^{-1} 3464,3340,2929,1710$ and 1691; ${ }^{1} \mathrm{H}$ NMR $\left(\mathrm{CDCl}_{3}, 400 \mathrm{MHz}\right) \delta 1.42(\mathrm{t}, 3 \mathrm{H}, J 6.0 \mathrm{~Hz})$, $4.43(\mathrm{q}, 2 \mathrm{H}, J 6.0 \mathrm{~Hz}), 7.33-7.51(\mathrm{~m}, 4 \mathrm{H}), 7.66-7.79(\mathrm{~m}$, $2 \mathrm{H}), 8.03(\mathrm{~d}, 1 \mathrm{H}, J 8.0 \mathrm{~Hz}), 9.92(\mathrm{br} \mathrm{s}, 1 \mathrm{H}) ;{ }^{13} \mathrm{C} \mathrm{NMR}$ $\left(\mathrm{CDCl}_{3}, 75 \mathrm{MHz}\right) \delta 161.4,135.2,130.1,129.0,128.6$, 127.7, 126.5, 124.8,124.1, 122.9, 121.6, 112.3, 109.1, 61.8, 14.2; MALDI-TOF MS m/z $239\left(\mathrm{M}^{+}\right)$; Anal. calcd. for $\mathrm{C}_{15} \mathrm{H}_{13} \mathrm{NO}_{2}$ : C 75.30, H 5.48, N 5.85; found: C 75.42, $\mathrm{H}$ 5.51, N 5.83.

Ethyl 6-chloro-3-methyl-1H-indole-2-carboxylate

White solid; mp 159-161 ${ }^{\circ} \mathrm{C}$ (lit. ${ }^{6} \mathrm{mp} 159-160{ }^{\circ} \mathrm{C}$ ); IR (KBr) $v_{\text {max }} / \mathrm{cm}^{-1} 3460,3339,2929,1710$ and 1691; ${ }^{1} \mathrm{H}$ NMR $\left(\mathrm{CDCl}_{3}, 400 \mathrm{MHz}\right) \delta 1.41(\mathrm{t}, 3 \mathrm{H}, J 8.0 \mathrm{~Hz})$, $2.59(\mathrm{~s}, 3 \mathrm{H}), 4.31(\mathrm{q}, 2 \mathrm{H}, J 8.0 \mathrm{~Hz}), 7.11-713(\mathrm{~m}, 1 \mathrm{H})$, 7.31-7.35 (d, 1H, J 1.8 Hz), 7.59 (d, 1H, J 9.6 Hz), 9.09 
(br s, $1 \mathrm{H}) ;{ }^{13} \mathrm{C}$ NMR $\left(\mathrm{CDCl}_{3}, 75 \mathrm{MHz}\right) \delta 161.7,137.2$, $130.1,128.4,125.6,122.5,121.3,120.5,110.2,61.3$, 14.9, 9.7; MALDI-TOF MS $m / z 237\left(\mathrm{M}^{+}\right)$; Anal. calcd. for $\mathrm{C}_{12} \mathrm{H}_{12} \mathrm{ClNO}_{2}$ : C 60.64, H 5.09, N 5.89; found: C 60.33, H 5.02, N 5.97.

\section{Ethyl 7-methoxyl-1H-indole-2-carboxylate}

White solid; mp 159-161 ${ }^{\circ} \mathrm{C}$ (lit. ${ }^{1} \mathrm{mp} 160-162{ }^{\circ} \mathrm{C}$ ); IR (KBr) $v_{\max } / \mathrm{cm}^{-1} 3465,3335,2930,1711$ and 1695; ${ }^{1} \mathrm{H} \mathrm{NMR}\left(\mathrm{CDCl}_{3}, 400 \mathrm{MHz}\right) \delta 1.39(\mathrm{t}, J 6.0 \mathrm{~Hz}, 3 \mathrm{H}), 3.83$ $(\mathrm{s}, 3 \mathrm{H}), 4.41(\mathrm{q}, 2 \mathrm{H}, J 6.0 \mathrm{~Hz}), 6.87(\mathrm{~s}, 1 \mathrm{H}), 7.01-7.09(\mathrm{~m}$, $1 \mathrm{H}), 7.25(\mathrm{~d}, 1 \mathrm{H}, J 2.0 \mathrm{~Hz}), 7.29(\mathrm{~d}, 1 \mathrm{H}, J 7.2 \mathrm{~Hz}), 9.11$ (br s, $1 \mathrm{H}) ;{ }^{13} \mathrm{C}$ NMR $\left(\mathrm{CDCl}_{3}, 75 \mathrm{MHz}\right) \delta 160.9,145.2$, 130.2, 129.1, 128.8, 122.3, 118.6, 111.0, 105.4, 61.7, 56.2, 14.4; MALDI-TOF MS m/z $219\left(\mathrm{M}^{+}\right)$; Anal. calcd. for $\mathrm{C}_{12} \mathrm{H}_{13} \mathrm{NO}_{3}$ : C 65.74, $\mathrm{H}$ 5.98, N 6.39; found: C 65.69, H 5.91, N 6.44.

\section{Ethyl 1H-pyrrolo[3,2-b]pyridine-2-carboxylate}

Brown solid; mp $179-181{ }^{\circ} \mathrm{C}$ (lit. ${ }^{7} \mathrm{mp} 179{ }^{\circ} \mathrm{C}$ ); IR (KBr) $v_{\text {max }} / \mathrm{cm}^{-1} 3405,3300,2887$ and 1685; ${ }^{1} \mathrm{H}$ NMR $\left(\mathrm{CDCl}_{3}, 400 \mathrm{MHz}\right) \delta 1.31(\mathrm{t}, 3 \mathrm{H}, J 7.2 \mathrm{~Hz}), 4.35(\mathrm{q}, 2 \mathrm{H}$, $J 7.2 \mathrm{~Hz}), 7.19(\mathrm{~d}, 1 \mathrm{H}, J 7.2 \mathrm{~Hz}), 7.27(\mathrm{dd}, 1 \mathrm{H}, J 4.8$, $8.1 \mathrm{~Hz}), 7.79(\mathrm{~d}, 1 \mathrm{H}, J 7.2 \mathrm{~Hz}), 8.37-8.41(\mathrm{~m}, 1 \mathrm{H}), 9.23(\mathrm{br}$ $\mathrm{s}, 1 \mathrm{H}) ;{ }^{13} \mathrm{C} \mathrm{NMR}\left(\mathrm{CDCl}_{3}, 75 \mathrm{MHz}\right) \delta 162.3,142.5,141.7$, 131.2, 130.1, 121.4, 120.6, 108.5, 61.1, 14.8; MALDI-TOF MS $m / z, 190\left(\mathrm{M}^{+}\right)$; Anal. calcd. for $\mathrm{C}_{10} \mathrm{H}_{10} \mathrm{~N}_{2} \mathrm{O}_{2}$ : C 63.15, $\mathrm{H}$ 5.30, N 14.73; found: C 63.01, H 5.37, N 14.81 .

\section{Ethyl thieno[3,2-b]pyrrole-5-carboxylate}

White solid; mp 132-134 ${ }^{\circ} \mathrm{C}$ (lit. ${ }^{8} \mathrm{mp} 132.5-133{ }^{\circ} \mathrm{C}$ ); IR (KBr) $v_{\text {max }} / \mathrm{cm}^{-1} 3400,3315,2893$ and 1693; ${ }^{1} \mathrm{H}$ NMR $\left(\mathrm{CDCl}_{3}, 400 \mathrm{MHz}\right) \delta 1.33(\mathrm{t}, 3 \mathrm{H}, J 7.1 \mathrm{~Hz}), 4.30(\mathrm{q}, 2 \mathrm{H}$, $J 7.1 \mathrm{~Hz}), 7.0(\mathrm{~d}, 1 \mathrm{H}, J 2.4 \mathrm{~Hz}), 7.18(\mathrm{~d}, 1 \mathrm{H}, J 2.4 \mathrm{~Hz})$, $7.26(\mathrm{~d}, 1 \mathrm{H}, J 3.2 \mathrm{~Hz}), 9.31$ (br s, $1 \mathrm{H}) ;{ }^{13} \mathrm{C} \mathrm{NMR}\left(\mathrm{CDCl}_{3}\right.$, $75 \mathrm{MHz}) \delta 162.1,131.7,130.0,125.1,122.4,118.3,109.2$, 61.7, 14.1; MALDI-TOF MS m/z $195\left(\mathrm{M}^{+}\right)$; Anal. calcd. for $\mathrm{C}_{9} \mathrm{H}_{9} \mathrm{NO}_{2} \mathrm{~S}$ : C 55.37, $\mathrm{H} 4.65, \mathrm{~N} 7.17$, S 16.42; found: C 55.41, H 4.57, N 7.21, S 16.33.
Ethyl 6-methyl-thieno[3,2-b]pyrrole-5-carboxylate

White solid; mp $144-146{ }^{\circ} \mathrm{C}$ (lit. ${ }^{8} \mathrm{mp} 144-145{ }^{\circ} \mathrm{C}$ ); IR (KBr) $v_{\max } / \mathrm{cm}^{-1}$ 3430, 3300, 2993 and 1687; ${ }^{1} \mathrm{H}$ NMR $\left(\mathrm{CDCl}_{3}, 400 \mathrm{MHz}\right) \delta 1.35(\mathrm{t}, 3 \mathrm{H}, J 7.2 \mathrm{~Hz}), 2.43(\mathrm{~s}, 3 \mathrm{H})$, $4.31(\mathrm{q}, 2 \mathrm{H}, J 7.2 \mathrm{~Hz}), 6.69(\mathrm{~d}, 1 \mathrm{H}, J 5.4 \mathrm{~Hz}), 7.31(\mathrm{~d}, 1 \mathrm{H}$, $J 5.4 \mathrm{~Hz}), 9.42$ (br s, $1 \mathrm{H}) ;{ }^{13} \mathrm{C}$ NMR $\left(\mathrm{CDCl}_{3}, 75 \mathrm{MHz}\right)$ $\delta 161.8,140.4,130.6,127.2,122.9,121.3,109.6,61.5$, 14.8, 11.7; MALDI-TOF MS m/z $209\left(\mathrm{M}^{+}\right)$; Anal. calcd. for $\mathrm{C}_{10} \mathrm{H}_{11} \mathrm{NO}_{2} \mathrm{~S}$ : C 57.39, H 5.30, N 6.69, S 15.32; found: C 57.27, H 5.38, N 6.57, S 15.26.

\section{Ethyl 2-nitrothieno[3,2-b]pyrrole-5-carboxylate}

Yellow solid; mp 187-189 ${ }^{\circ} \mathrm{C}$ (lit. ${ }^{9} \mathrm{mp} 188-189{ }^{\circ} \mathrm{C}$ ); IR (KBr) $v_{\max } / \mathrm{cm}^{-1} 3410,3303,1689,1490$ and 1370; ${ }^{1} \mathrm{H} \mathrm{NMR}\left(\mathrm{CDCl}_{3}, 400 \mathrm{MHz}\right) \delta 1.40(\mathrm{t}, 3 \mathrm{H}, J 7.0 \mathrm{~Hz}), 4.33(\mathrm{q}$, $2 \mathrm{H}, J 7.0 \mathrm{~Hz}), 7.11(\mathrm{~m}, 1 \mathrm{H}), 7.76(\mathrm{~d}, 1 \mathrm{H}, J 4.8 \mathrm{~Hz}), 9.39(\mathrm{br}$ $\mathrm{s}, 1 \mathrm{H}) ;{ }^{13} \mathrm{C} \mathrm{NMR}\left(\mathrm{CDCl}_{3}, 75 \mathrm{MHz}\right) \delta 162.7,151.3,137.4$, 131.6, 128.1, 122.2, 108.5, 60.6, 14.1; MALDI-TOF MS $\mathrm{m} / \mathrm{z} 240\left(\mathrm{M}^{+}\right)$; Anal. calcd. for $\mathrm{C}_{9} \mathrm{H}_{8} \mathrm{~N}_{2} \mathrm{O}_{4} \mathrm{~S}: \mathrm{C} 45.00, \mathrm{H} 3.36$, N 11.66, S 13.35; found: C 45.06, H 3.38, N 11.59, S 13.29.

\section{References}

1. Sanz, R.; Escribano, J.; Pedrosa, M. R.; Aguado, R.; Arnáiz, F. J.; Adv. Synth. Catal. 2007, 349, 713.

2. Acheson, R. M.; Prince, R. J.; Procter, G.; J. Chem. Soc., Perkin Trans. 1 1979, 3, 595.

3. Ning, Z. L.; Yang, F. L.; He, L. P.; Guo, Y.; Wang, W.; Li, G. Y.; Wang, X.; Wang, E. S.; Jilin Daxue Xuebao, Lixueban 2006, 1, 118; CA 2006, 146: 295872.

4. Gan, T.; Liu, R.; Yu, P.; Zhao, S.; Cook, J. M.; J. Org. Chem. 1997, 62, 9298.

5. Goldsmith, E. A.; Lindwall, H. G.; J. Org. Chem. 1953, 18, 507.

6. Kim, Y. A.; Han, S. Y.; Synth. Commun. 2004, 34, 2931.

7. Frydman, B.; Reil, S. J.; Boned, J.; Rapoport, H.; J. Org. Chem. 1968, 33, 3762.

8. Gale, W. W.; Scott, A. N.; Snyder, H. R.; J. Org. Chem. 1964, 29,2160

9. Gálvez, J. E. C.; García, F.; J. Heterocycl. Chem. 1984, 21, 215. 


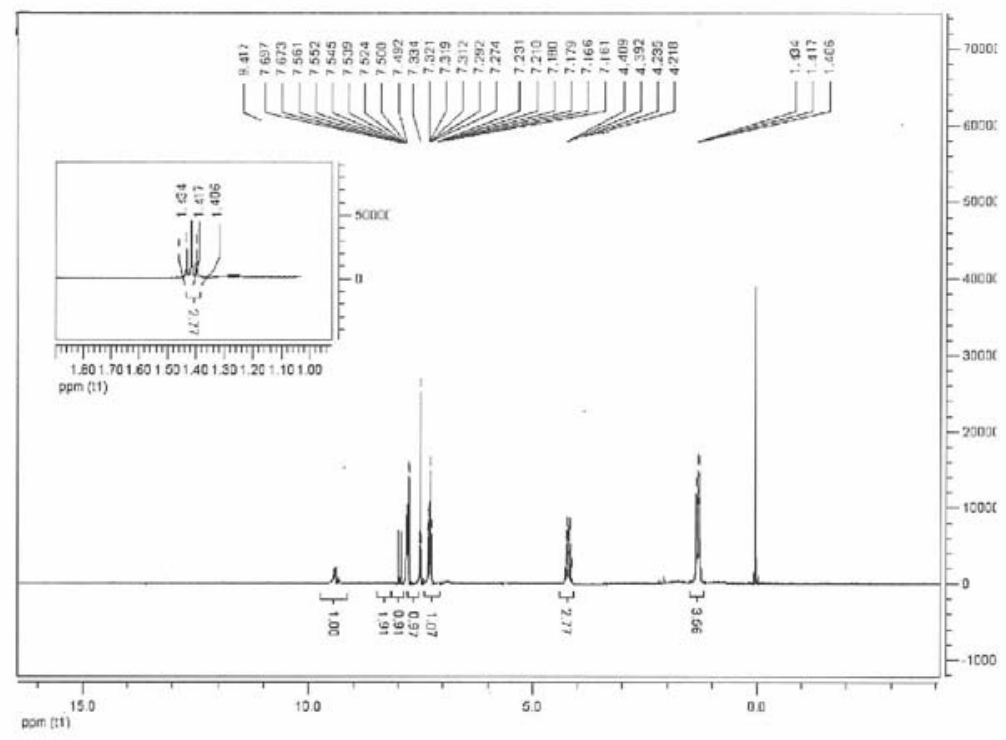

Figure S1. ${ }^{1} \mathrm{H}$ NMR spectrum $\left(\mathrm{CDCl}_{3}, 400 \mathrm{MHz}\right)$ of ethyl $1 \mathrm{H}$-indole-2-carboxylate.

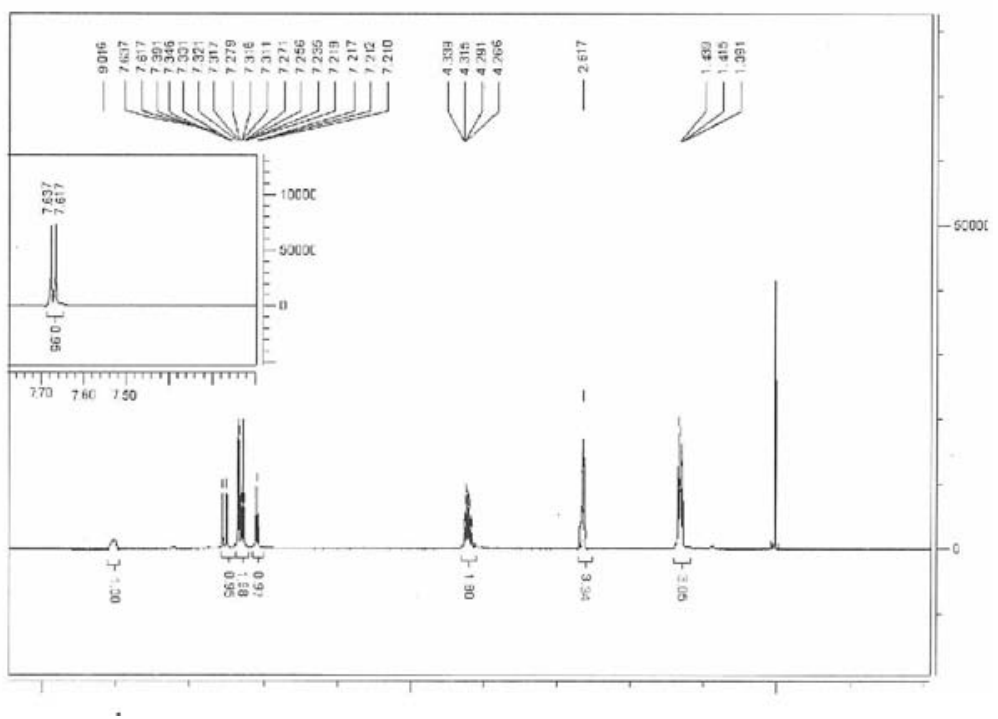

Figure S2. ${ }^{1} \mathrm{H}$ NMR spectrum $\left(\mathrm{CDCl}_{3}, 400 \mathrm{MHz}\right)$ of ethyl 3-methyl- $1 H$-indole-2-carboxylate. 


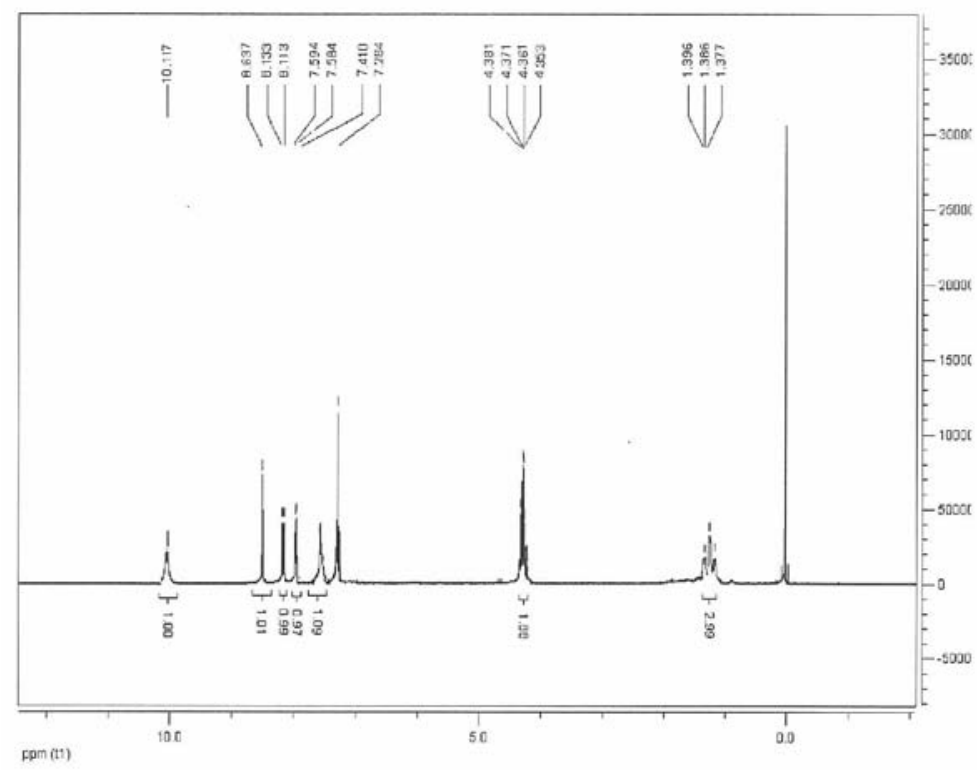

Figure S3. ${ }^{1} \mathrm{H}$ NMR spectrum $\left(\mathrm{CDCl}_{3}, 400 \mathrm{MHz}\right)$ of ethyl 5-nitrol-1H-indole-2-carboxylate.

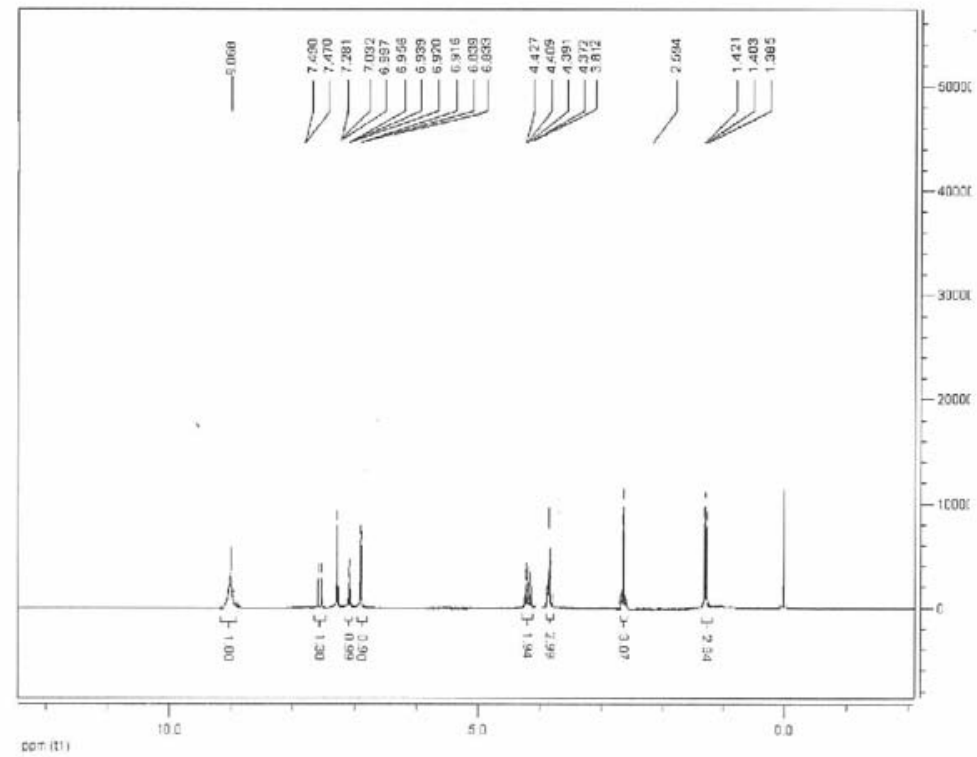

Figure S4. ${ }^{1} \mathrm{H}$ NMR spectrum $\left(\mathrm{CDCl}_{3}, 400 \mathrm{MHz}\right)$ of ethyl 6-methoxyl-3-methyl-1H-indole-2-carboxylate. 


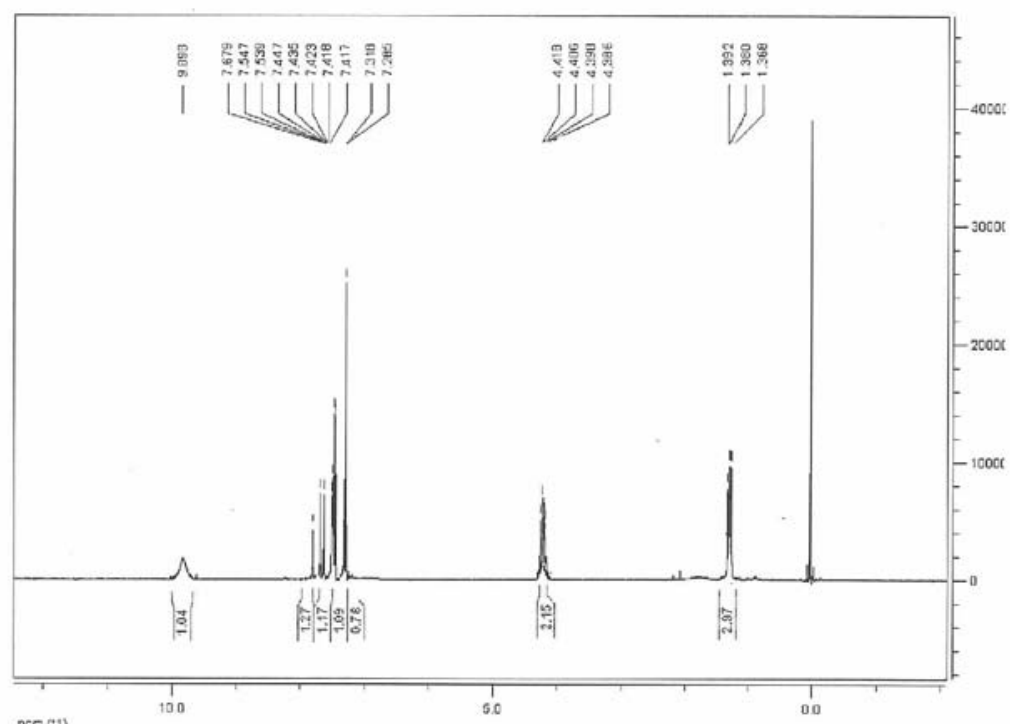

Figure S5. ${ }^{1} \mathrm{H}$ NMR spectrum $\left(\mathrm{CDCl}_{3}, 400 \mathrm{MHz}\right)$ of ethyl 5-chloro-1H-indole-2-carboxylate.

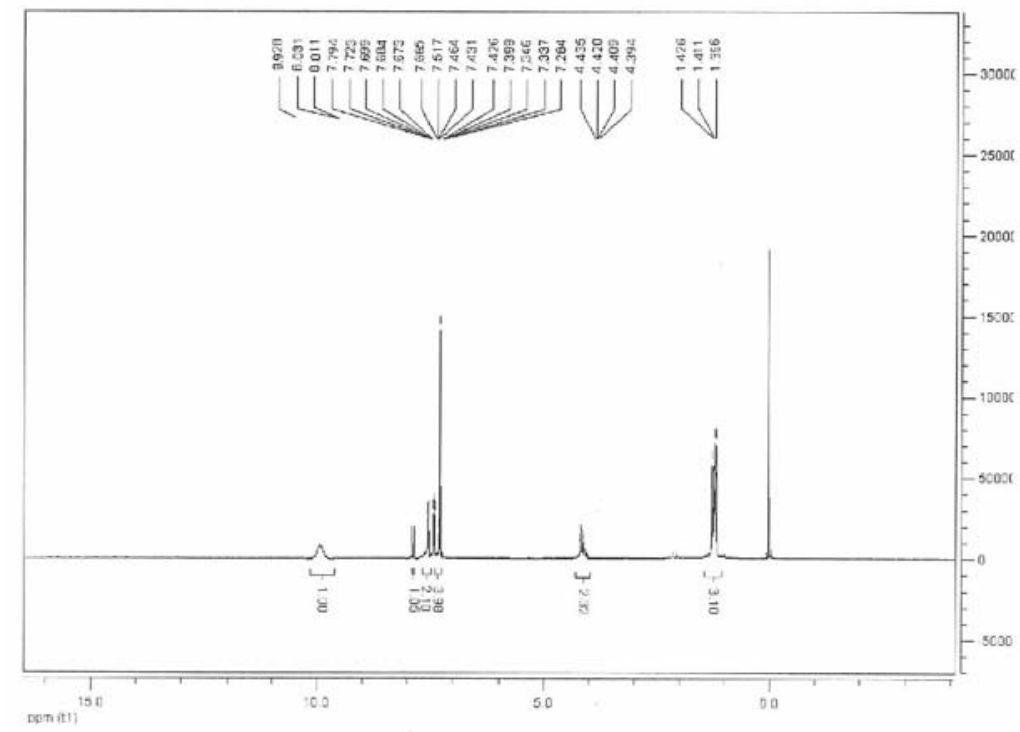

Figure S6. ${ }^{1} \mathrm{H}$ NMR spectrum $\left(\mathrm{CDCl}_{3}, 400 \mathrm{MHz}\right)$ of ethyl $3 \mathrm{H}$-benz[e]indole-2-carboxylate. 


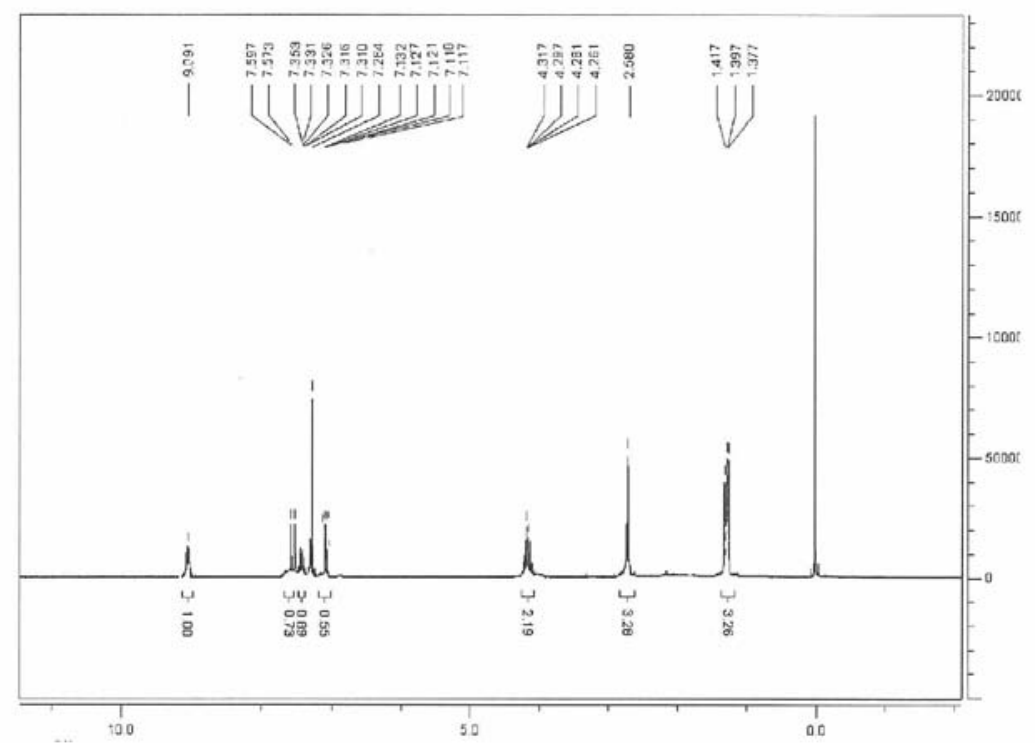

Figure S7. ${ }^{1} \mathrm{H}$ NMR spectrum $\left(\mathrm{CDCl}_{3}, 400 \mathrm{MHz}\right)$ of ethyl 6-chloro-3-methyl-1H-indole-2-carboxylate.

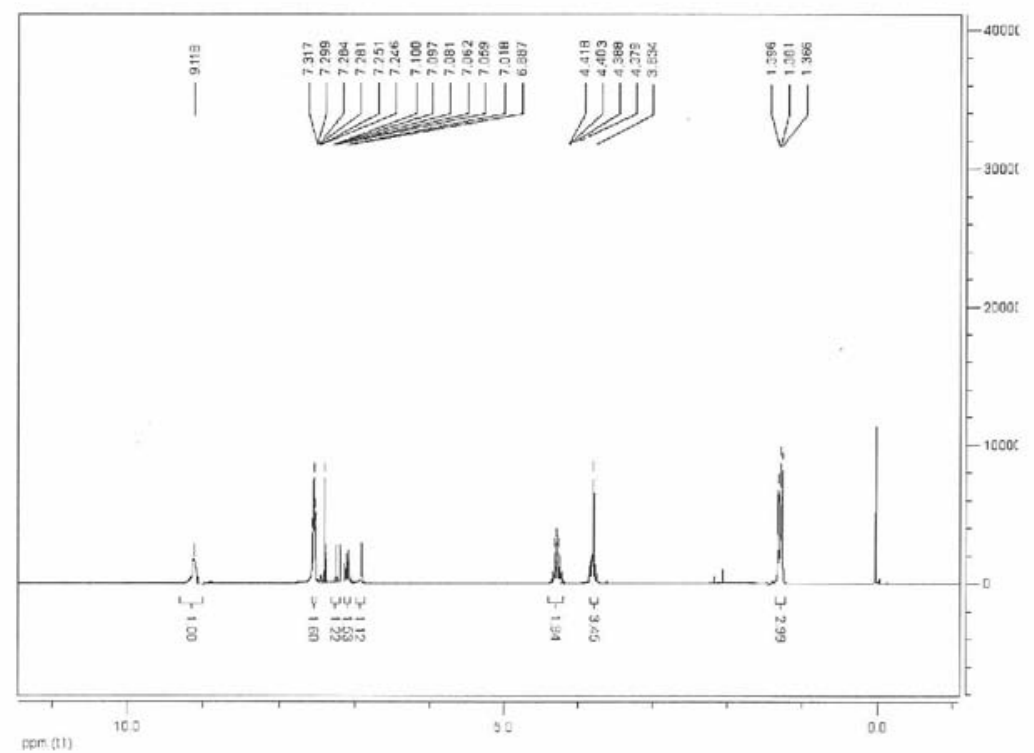

Figure S8. ${ }^{1} \mathrm{H}$ NMR spectrum $\left(\mathrm{CDCl}_{3}, 400 \mathrm{MHz}\right)$ of ethyl 7-methoxyl-1H-indole-2-carboxylate. 


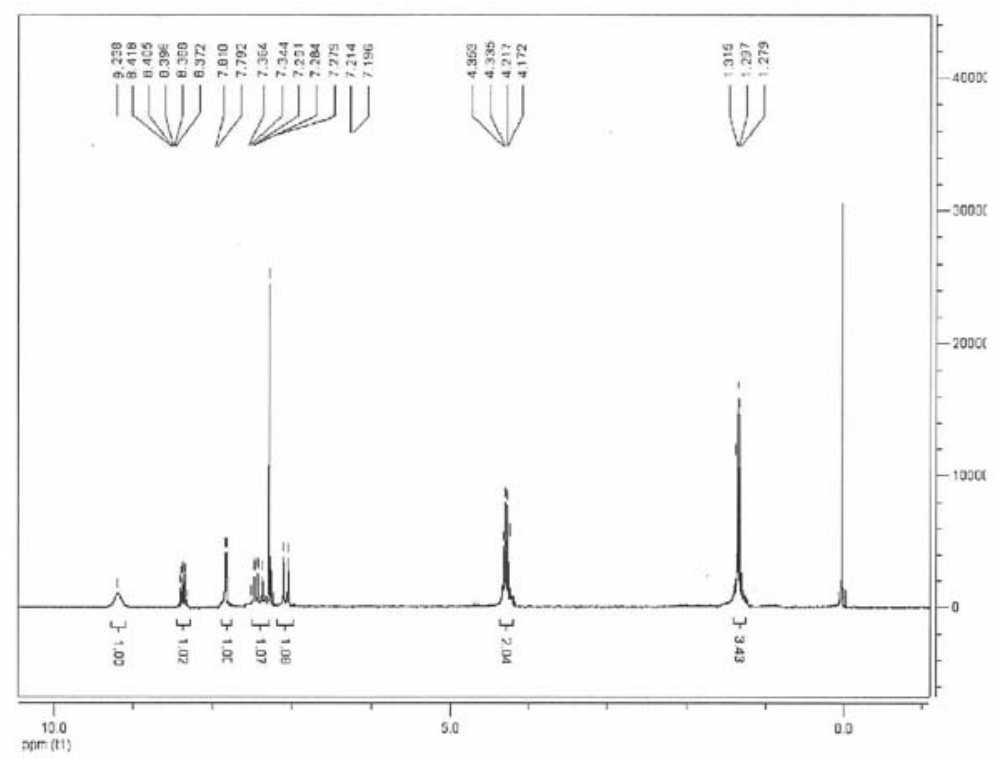

Figure S9. ${ }^{1} \mathrm{H}$ NMR spectrum $\left(\mathrm{CDCl}_{3}, 400 \mathrm{MHz}\right)$ of ethyl $1 \mathrm{H}$-pyrrolo[3,2-b]pyridine-2-carboxylate.

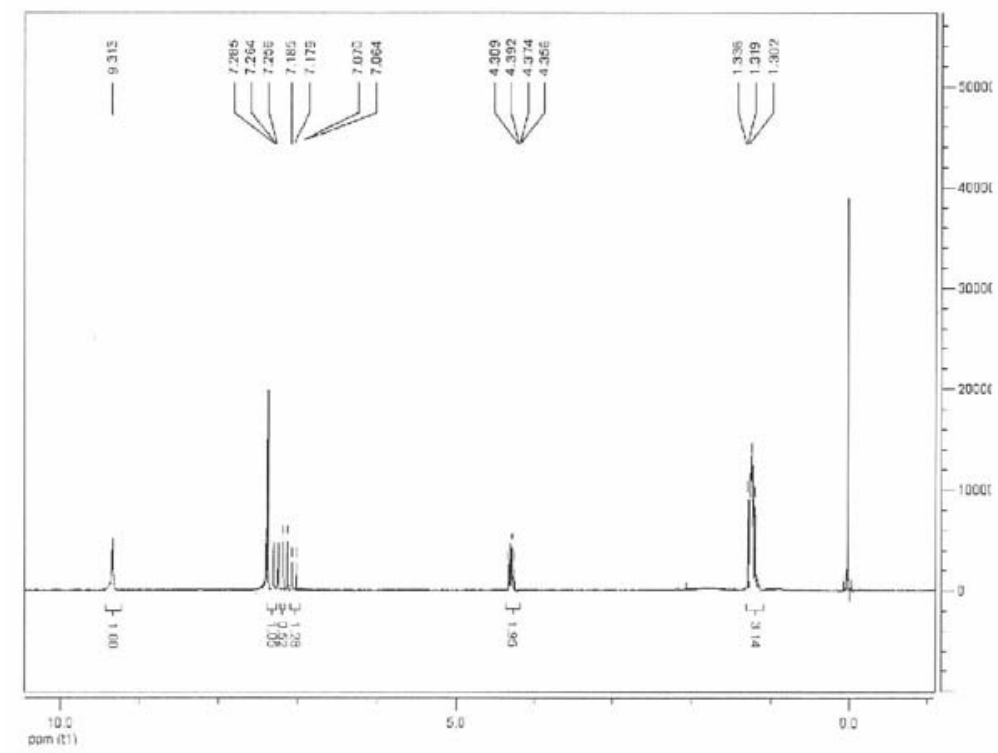

Figure S10. ${ }^{1} \mathrm{H}$ NMR spectrum $\left(\mathrm{CDCl}_{3}, 400 \mathrm{MHz}\right)$ of ethyl thieno[3,2-b]pyridine-5-carboxylate. 


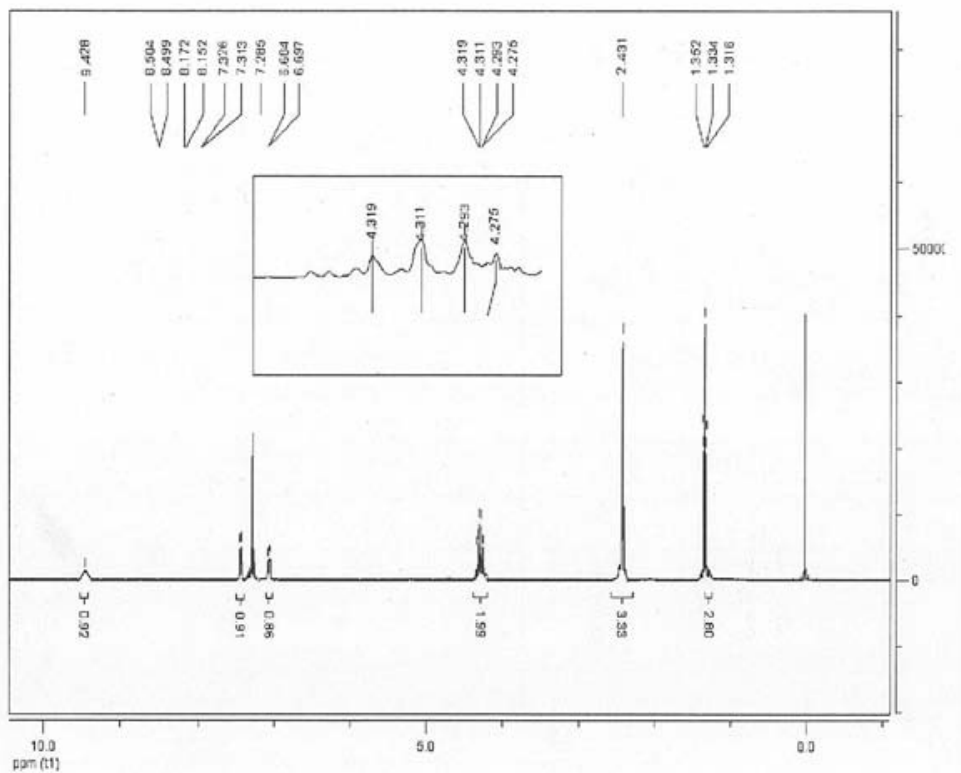

Figure S11. ${ }^{1} \mathrm{H}$ NMR spectrum $\left(\mathrm{CDCl}_{3}, 400 \mathrm{MHz}\right)$ of ethyl 6-methyl-thieno[3,2-b]pyrrole-5-carboxylate.

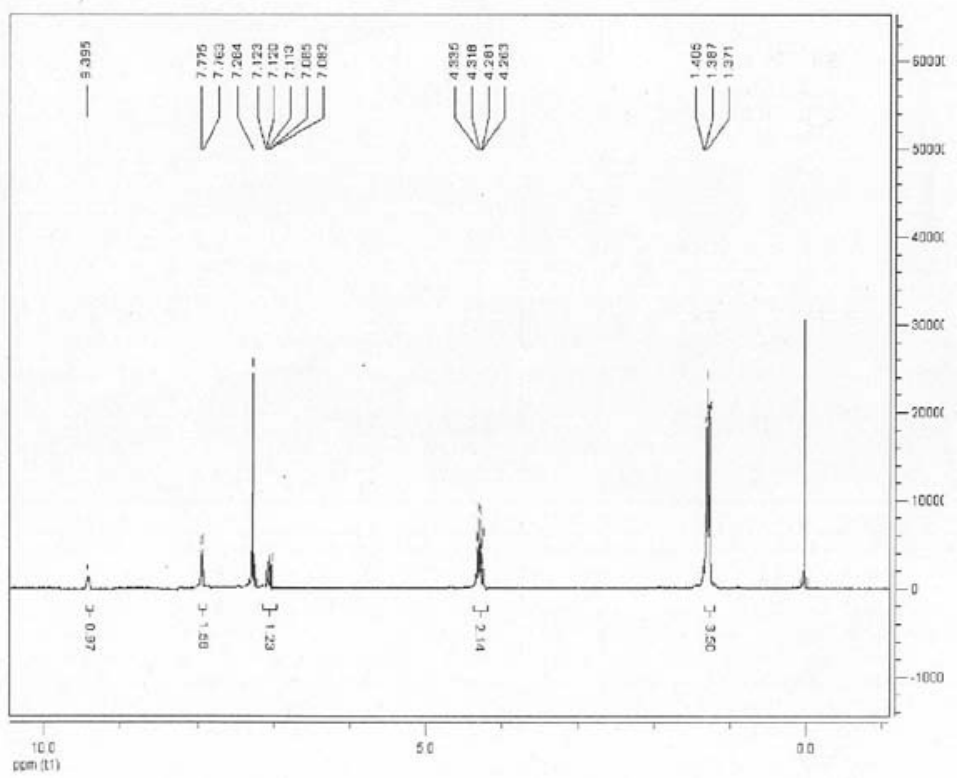

Figure S12. ${ }^{1} \mathrm{H}$ NMR spectrum $\left(\mathrm{CDCl}_{3}, 400 \mathrm{MHz}\right)$ of ethyl 2-nitrothieno[3,2-b]pyrrole-5-carboxylate. 


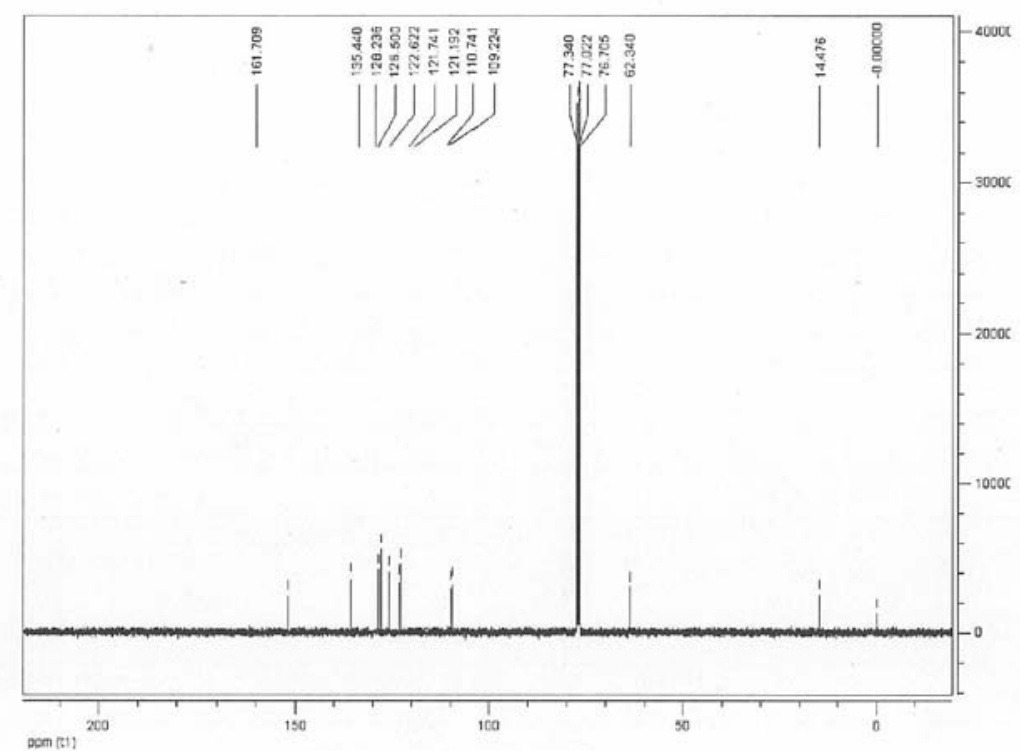

Figure S13. ${ }^{13} \mathrm{C}$ NMR spectrum $\left(\mathrm{CDCl}_{3}, 75 \mathrm{MHz}\right)$ of ethyl $1 H$-indole-2-carboxylate.

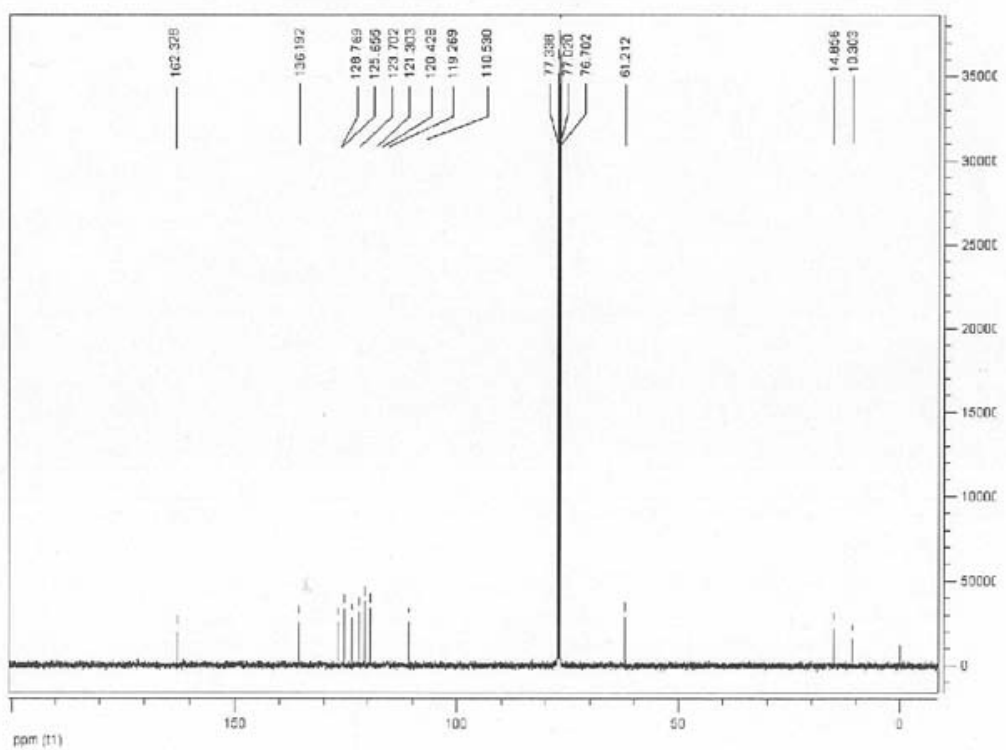

Figure S14. ${ }^{13} \mathrm{C}$ NMR spectrum $\left(\mathrm{CDCl}_{3}, 75 \mathrm{MHz}\right)$ of ethyl 3-methyl- $1 H$-indole-2-carboxylate. 


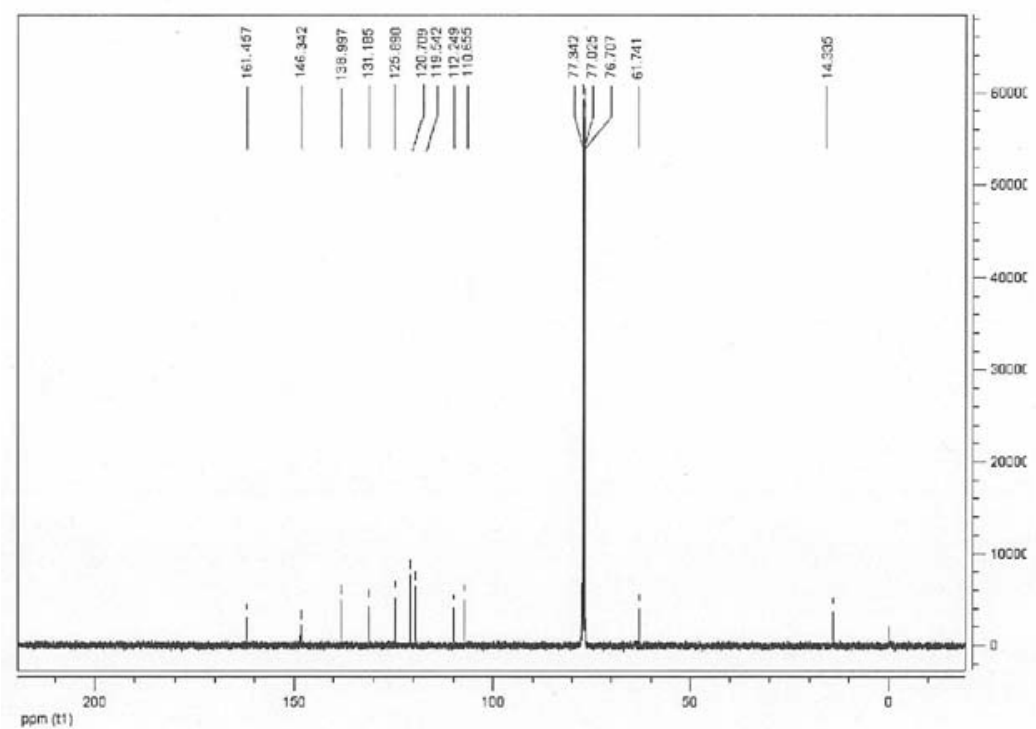

Figure S15. ${ }^{13} \mathrm{C}$ NMR spectrum $\left(\mathrm{CDCl}_{3}, 75 \mathrm{MHz}\right)$ of ethyl 5-nitrol-1H-indole-2-carboxylate.

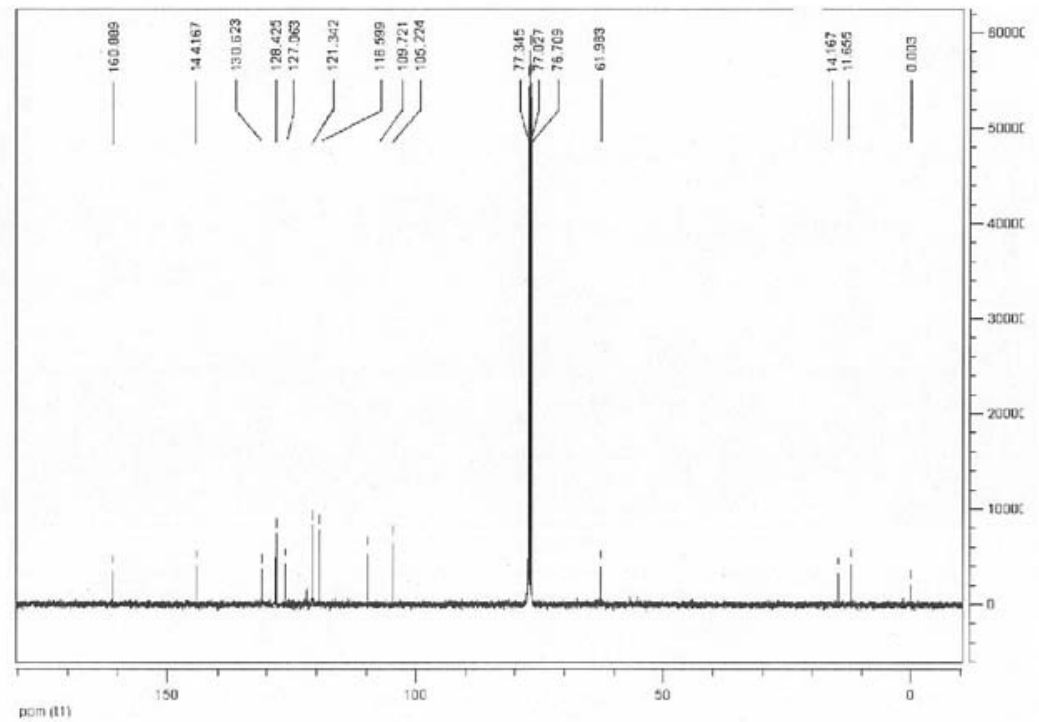

Figure S16. ${ }^{13} \mathrm{C}$ NMR spectrum $\left(\mathrm{CDCl}_{3}, 75 \mathrm{MHz}\right)$ of ethyl 6-methoxyl-3-methyl-1 $H$-indole-2-carboxylate. 


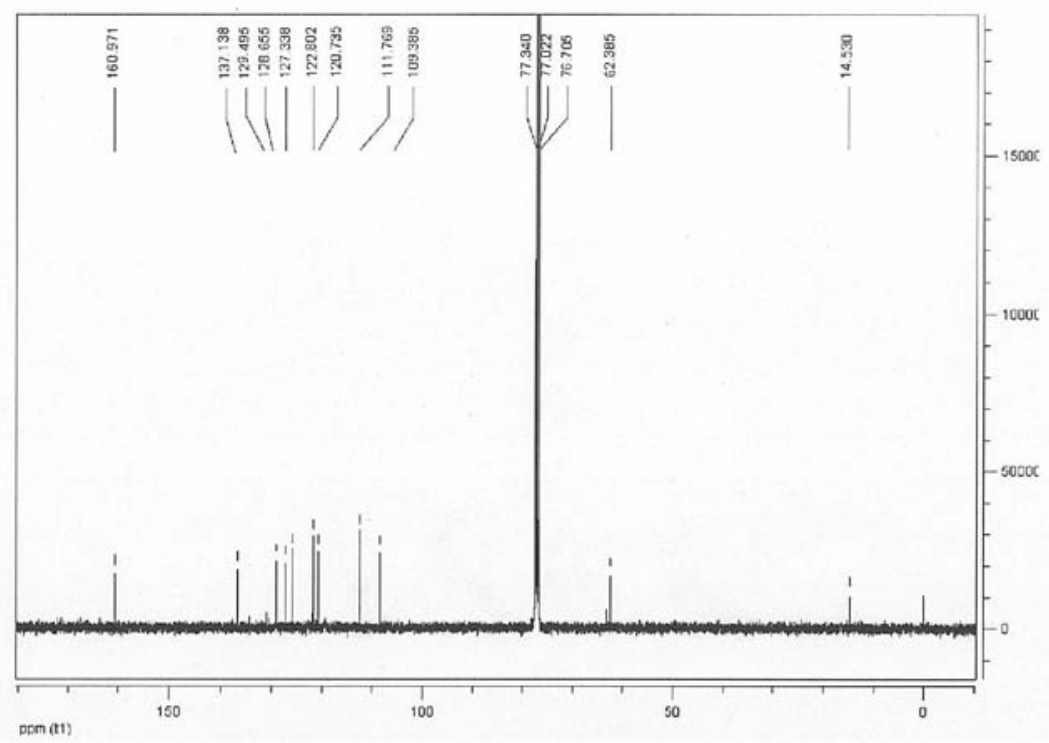

Figure S17. ${ }^{13} \mathrm{C}$ NMR spectrum $\left(\mathrm{CDCl}_{3}, 75 \mathrm{MHz}\right)$ of ethyl 5-chloro- $1 \mathrm{H}$-indole-2-carboxylate.

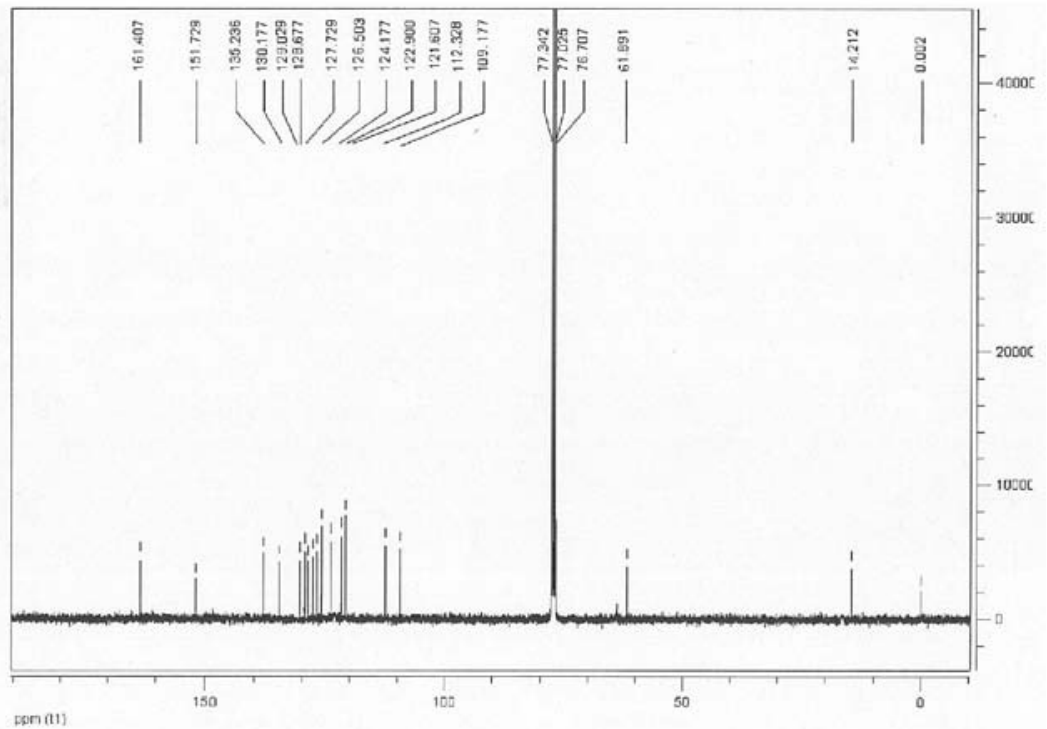

Figure S18. ${ }^{13} \mathrm{C}$ NMR spectrum $\left(\mathrm{CDCl}_{3}, 75 \mathrm{MHz}\right)$ of ethyl $1 \mathrm{H}$-benz[e]indole-2-carboxylate. 


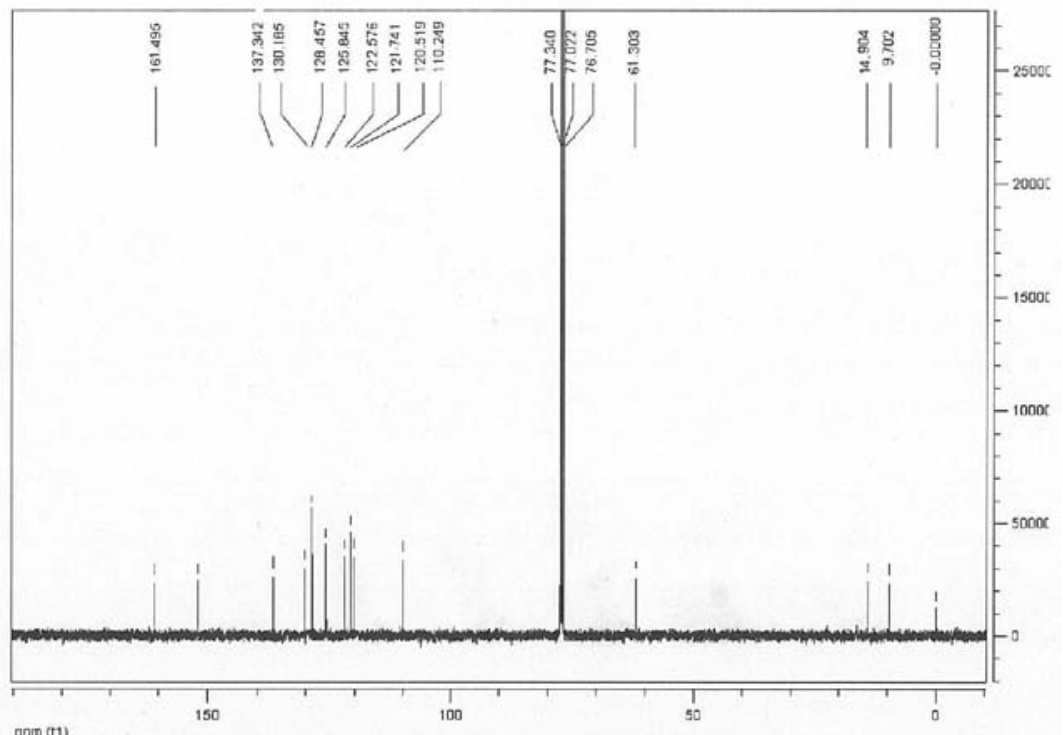

Figure S19. ${ }^{13} \mathrm{C}$ NMR spectrum $\left(\mathrm{CDCl}_{3}, 75 \mathrm{MHz}\right)$ of ethyl 6 chloro-3-methyl-1H-indole-2-carboxylate.

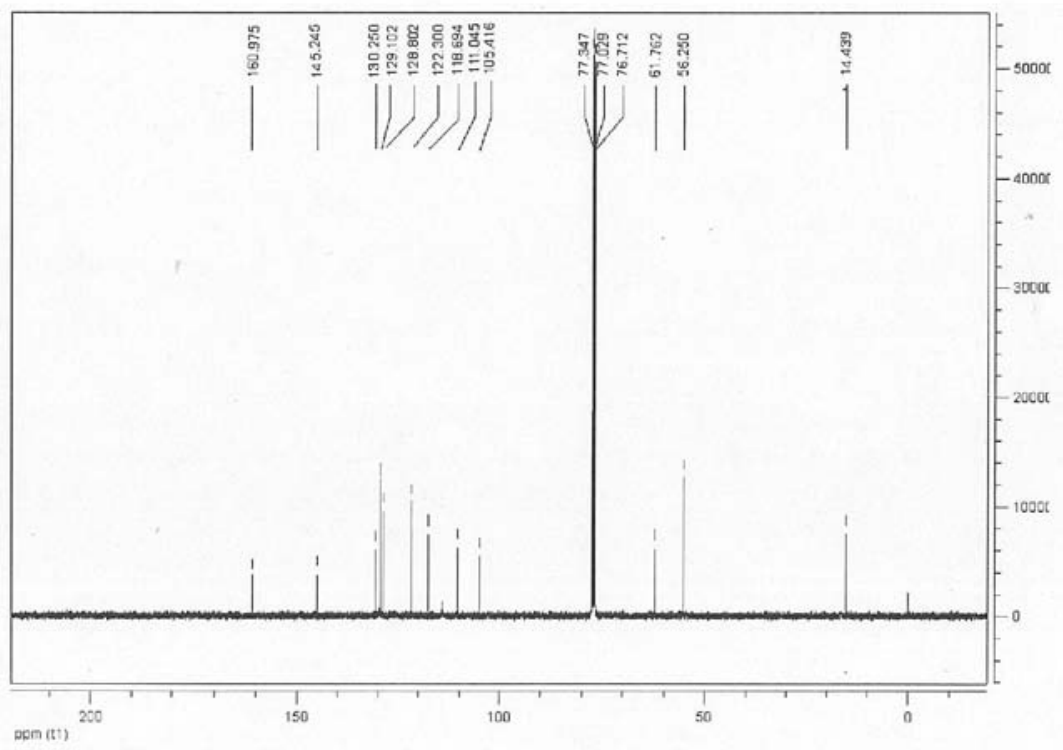

Figure S20. ${ }^{13} \mathrm{C}$ NMR spectrum $\left(\mathrm{CDCl}_{3}, 75 \mathrm{MHz}\right)$ of ethyl 7-methoxyl- $1 \mathrm{H}$-indole-2-carboxylate. 


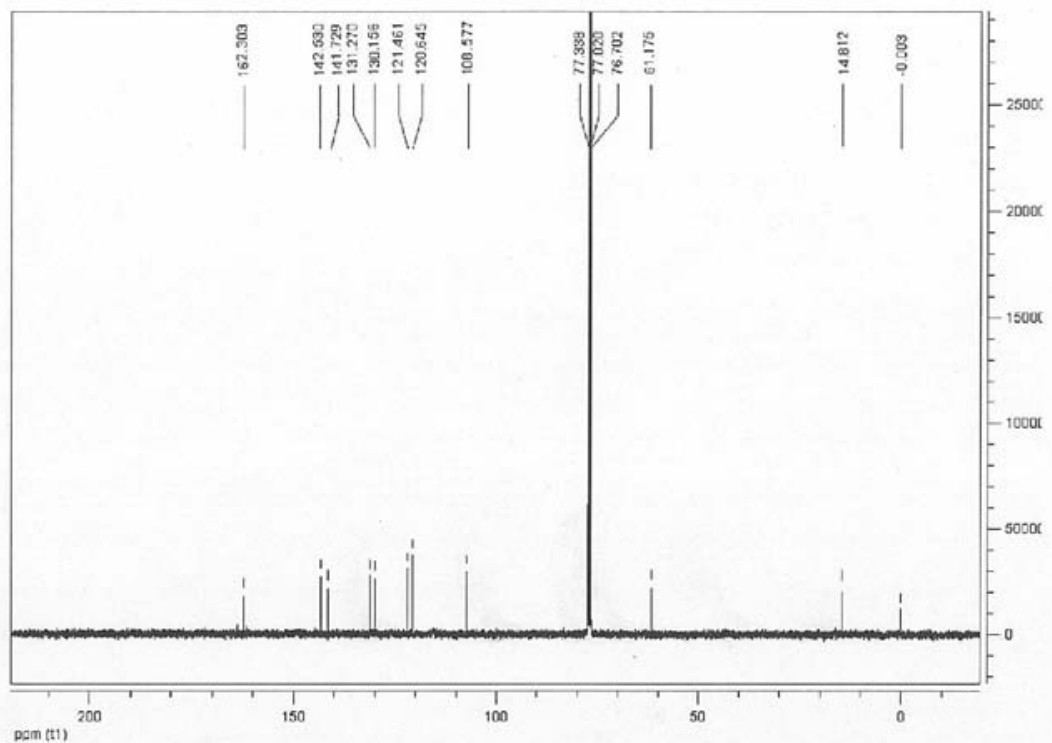

Figure S21. ${ }^{13} \mathrm{C}$ NMR spectrum $\left(\mathrm{CDCl}_{3}, 75 \mathrm{MHz}\right)$ of ethyl $1 \mathrm{H}$-pyrrolo[3,2-b]pyridine-2-carboxylate.

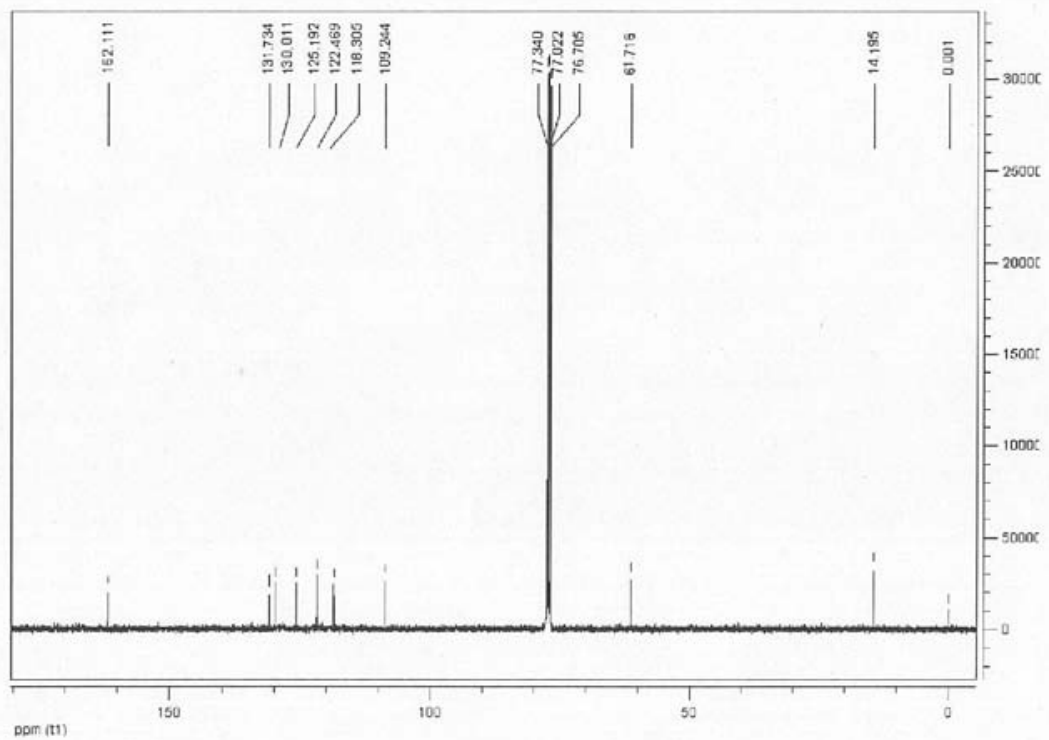

Figure S22. ${ }^{13} \mathrm{C}$ NMR spectrum $\left(\mathrm{CDCl}_{3}, 75 \mathrm{MHz}\right)$ of ethyl thieno[3,2-b]pyrrole-5-carboxylate. 


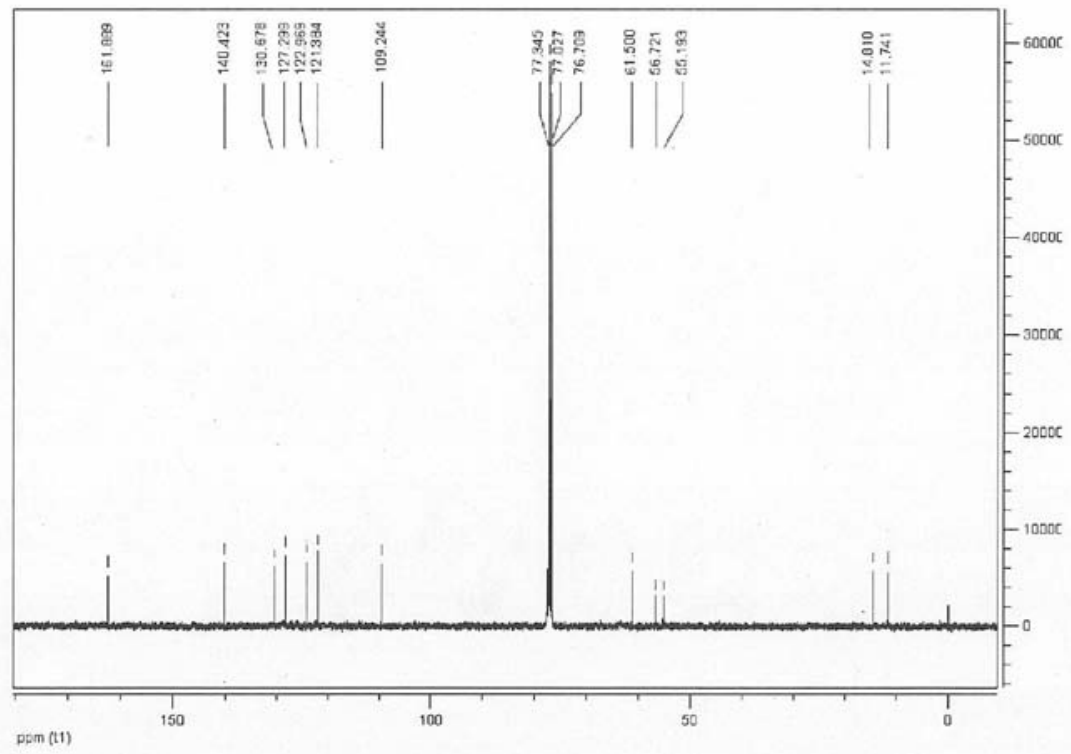

Figure S23. ${ }^{13} \mathrm{C}$ NMR spectrum $\left(\mathrm{CDCl}_{3}, 75 \mathrm{MHz}\right)$ of ethyl 6-methyl-thieno[3,2-b]pyrrole-5-carboxylate.

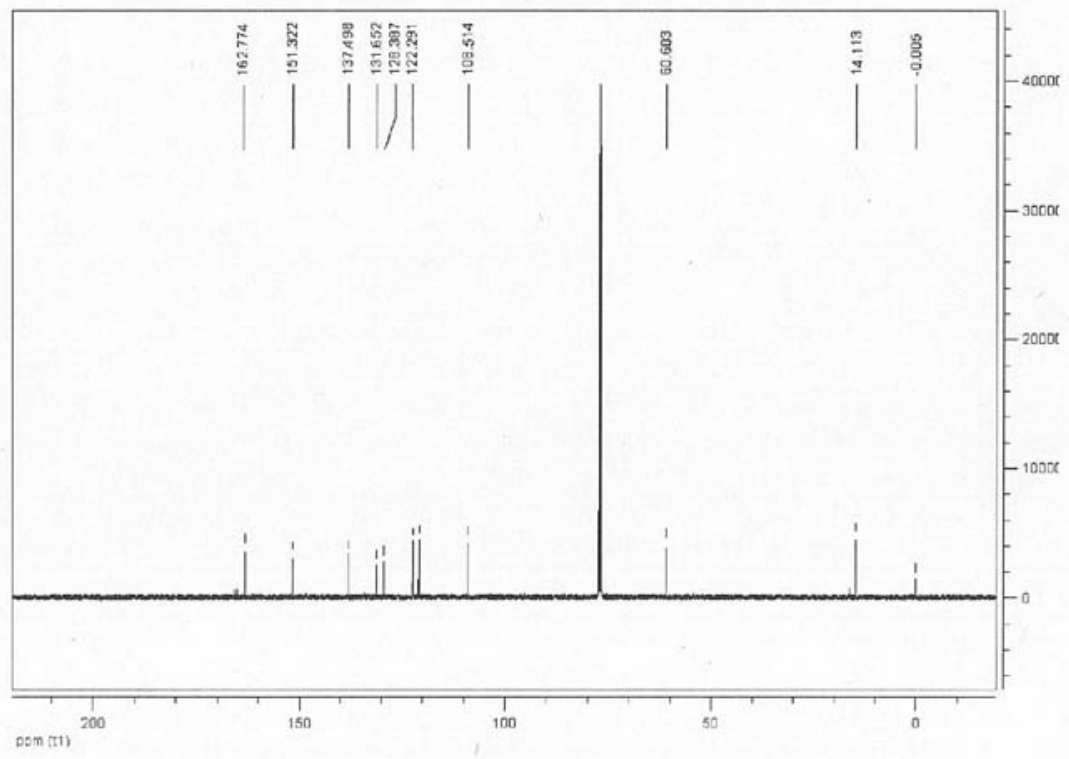

Figure S24. ${ }^{13} \mathrm{C}$ NMR spectrum $\left(\mathrm{CDCl}_{3}, 75 \mathrm{MHz}\right)$ of ethyl 2-nitrothieno[3,2-b]pyrrole-5-carboxylate. 